\title{
SILENT TRANSMISSION OF THE DENGUE VIRUS IN SOUTHERN TAIWAN
}

\author{
WEI-JUNE CHEN, SU-LIH CHEN, LI-JUNG CHIEN, CHI-CHEN CHEN, \\ CHWAN-CHUEN KING, MING-RONG HARN, KAO-PIN HWANG, AND JIH-HUA FANG \\ Department of Parasitology, Chang Gung College of Medicine and Technology, Kwei-San, Tao- \\ Yuan, Taiwan
}

\begin{abstract}
Immunoglobulin M (IgM) antibody to dengue virus was examined from a total of 3,099 serum samples collected in southern Taiwan. Of 1,232 sera collected from a junior high school and four elementary schools in LiuChiu, 35 were IgM-positive, demonstrating that the dengue virus has been circulating on the island, despite the fact that no epidemic has been reported in the past 10 years. Sixteen of 925 sera collected from three elementary schools in Tung-Kang in 1991 were found to be IgM-positive and two of 192 sera from adults in the local community were positive. The IgM-positive subjects tended to be aggregated around a port. Fishing boats that had stopped in neighboring endemic countries were presumed to have introduced the virus periodically, causing a low level of inapparent infections. In the Kaohsiung area, two of 108 suspected clinical cases and four of 642 community-based sera were IgM-positive. Rapid urbanization has provided appropriate circumstances for vector breeding in this area and the high population density has also increased contact frequency between humans and mosquito vectors. This has, in turn, increased the possibility of silent transmission of the dengue virus via either intermittent reintroduction of the virus or continuation of inapparent infections or both. Establishment of a early warning system using the IgM antibody capture-enzyme-linked immunosorbent assay is suggested for effective monitoring of the disease.
\end{abstract}

Dengue fever has been seen as an important public health problem in southeast Asia and Latin America. ${ }^{1.2}$ In Taiwan, no outbreak was reported after 1945 until an epidemic occurred on Liu-Chiu island in 1981 , resulting in $80 \%$ of the population becoming infected.34 Another major epidemic, primarily caused by dengue 1 virus, occurred from late 1987 through 1988 in southern Taiwan. ${ }^{5}$ Since then, sporadic cases have occasionally been reported. ${ }^{6,7}$ Recently, traffic between Taiwan and neighboring countries, either by sea or by air, has been quite busy. In addition, there have been more than 100,000 non-Taiwanese southeast Asians working annually in Taiwan since 1992, increasing the possibility of dengue being introduced.

Dengue fever is transmitted by Aedes mosquitoes among susceptible human hosts. ${ }^{8}$ In southern Taiwan the Breteau index has remained $>5,9$ making it difficult to avoid an outbreak should the virus be introduced. Moreover, silent transmission of dengue infections has recently been recognized. For the purpose of this study, silent transmission refers to inapparent or asymptomatic with dengue virus. These infections are common in Taiwan. ${ }^{10}$ Therefore, a long-termed active surveillance has become necessary in any place at risk.

For infected patients, dengue virus immunoglobulin $\mathbf{M}$ (IgM) antibodies usually appear earlier than IgG antibodies and do not decrease until 30-60 days later. ${ }^{11,12}$ Thus, an assay detecting IgM antibody was recently used to confirm flaviviral infections such as Japanese encephalitis (JE) virus $^{13}$ and dengue virus, ${ }^{14}$ for which IgM antibody detection has become a useful technique for diagnosis ${ }^{15.16}$ and epidemiologic surveys. ${ }^{17}$ Recently, an enzyme-linked immunosorbent assay (ELISA) has been used extensively for the detection of IgM antibody due to the rapidity, high sensitivity, and high specificity of this technique. ${ }^{14}$ As a result, we have applied the IgM antibody-capture ELISA (MAC-ELISA) to investigate whether or not transmission of dengue virus is continuing in southern Taiwan. Ideally, the MAC-ELISA can also be a good warning system to provide information that will help prevent an outbreak from spreading. ${ }^{18}$

\section{MATERIALS AND METHODS}

Collection of serum specimens. This study covered two cities, Kaohsiung and Ping-Tung. Three districts of Kaohsiung, Feng-San, Kang-San, and San-Min, and two in PingTung, Liu-Chiu and Tung-Kang, were included. All study sites are located in the southern part of Taiwan. Of them, Liu-Chiu is a small island about $15 \mathrm{~km}$ from Tung-Kang.

A total of 642 serum specimens were collected from residents in July-September 1992, from the Kaohsiung area, including 203 in Kang-San, 234 in Feng-San, and 205 in San-Min. In addition, clinical samples $(n=108)$ were collected from suspected cases in Harn's Clinic and Kaohsiung Medical College Hospital in 1991-1993 (January-July).

The survey in Liu-Chiu was based on 1,232 serum samples collected from four elementary schools and one junior high school in January-February 1990. Liu-Chiu island was geographically divided into eight villages. Serum samples of Tung-Kang were collected from three elementary schools. A total of 925 blood samples were taken from school children in January-February 1991, and another 192 sera were collected from the community in July-September 1992.

Cell culture and antigen preparation. The $\mathrm{C} 6 / 36$ cells used in the present study were maintained in Eagle's minimal essential medium supplemented with $10 \%$ fetal bovine serum at $28^{\circ} \mathrm{C}$. Four prototype dengue viruses (Hawaii, New Guinea C, H87, and H241), which were obtained from the Centers for Disease Control and Prevention San Juan Laboratory in Puerto Rico, were used for antigen production. To prepare antigen, confluent cells were inoculated with viruses at a multiplicity of infection (number of infectious virus particles/number of cells inoculated) of 0.1-1. Inoculated cells were then harvested to verify the infection using a direct immunofluorescence antibody test after an incubation period of seven days. The cells were subsequently frozen and thawed several times and centrifuged at 3,000 rpm for 15 min at $4^{\circ} \mathrm{C}$. Discarded cell debris and the supernatant, which would be used as the antigen, was stored at $-70^{\circ} \mathrm{C}$ until use. 
The antigen for the assay was prepared from the cell culture fluid, and its final dilution was based on the virus titer in the supernatant. The working antigen preparation was actually a mixture of the four prototypes of the virus.

Immunoglobulin $M$ capture ELISA. The MAC-ELISA for IgM detection followed the method described by Kuno and others. ${ }^{19}$ Briefly, Linbro microplates (Flow Laboratories, McLean, VA) were coated with $100 \mu$ l of goat anti-human IgM diluted to 1:200 with $0.1 \mathrm{M}$ carbonate buffer. The coated plates were stored at $4^{\circ} \mathrm{C}$ until use. Before testing the specimen, the plates were washed with phosphate-buffered saline (PBS, pH 7.4). Four percent bovine serum albumin (BSA) was used for the blocking reaction. The plates were incubated for $15 \mathrm{~min}$ at $37^{\circ} \mathrm{C}$ and washed five times with PBS. Then $0.05 \mathrm{ml}$ of the serum specimen diluted to $1: 10$ in $0.05 \%$ BSA-PBS was added and the plates were incubated at room temperature for $2 \mathrm{hr}$. They were then washed again and $0.5 \mathrm{ml}$ the prepared antigen was added. After overnight incubation at $4^{\circ} \mathrm{C}$, they were washed again and $0.025 \mathrm{ml}$ of horseradish peroxidase-conjugated 6B6C-1 monoclonal antibody (kindly provided by Dr. D. J. Gubler, Centers for Disease Control and Prevention, Fort Collins, CO) diluted 1: 6,000 in $20 \%$ normal human serum was added to each well. The plates were then incubated at $37^{\circ} \mathrm{C}$ for $1 \mathrm{hr}$, washed again, and $0.1 \mathrm{ml}$ of ABTS (Kirkegaard and Perry Laboratories, Inc, Gaithersburg, VA) substrate solution was added to each well. After incubation at $37^{\circ} \mathrm{C}$ for $30 \mathrm{~min}$ and standing at room temperature for $2 \mathrm{hr}$, the optical density (OD) value was read with the Anthos (Salzburg, Austria) htll ELI$\mathrm{SA}$ reader at a wavelength of $405 \mathrm{nmn}$.

Evaluation of ELISA reults. For quality control, one positive control and three negative controls were always included in each plate. The positive designation was made when the OD value of the sample was higher than the average of three negative controls plus three standard errors. All data related to IgM prevalence rates were analyzed using the chi-square test.

Direct immunofiuorescence antibody (DFA) test. The DFA technique followed the method described by Kuberski and Rosen. ${ }^{20}$ Briefly, harvested cells were smeared on a teflon-coated slide. After fixation in cold acetone, a drop of fluorescein isothiocyanate-conjugated antibody against the dengue virus from human hyperimmune serum was added to the cells. The smears were then incubated at $37^{\circ} \mathrm{C}$ for 30 min in a humid chamber, washed in PBS for $10 \mathrm{~min}$, and mounted with a mixture of glycerol and PBS (1:9). They were then examined under a fluorescence microscope (Optiphot-2; Nikon, Tokyo, Japan). Positive and negative controls were included in each experiment.

Hemagglutination inhibition (HI) test. This test followed the procedure described by Clarke and Casals. ${ }^{21}$ Briefly, $0.025 \mathrm{ml}$ of acetone-extracted serum was added to each well of a 96-well U-shaped plate. Two-fold serial dilutions were made with $0.4 \%$ bovine albumin in borate saline, and $0.025 \mathrm{ml}$ of antigen ( 8 hemagglutination units) (acetone-extracted from mouse brain) was added to each well, mixed thoroughly, then incubated at $4^{\circ} \mathrm{C}$ for $18-21 \mathrm{hr}$. Finally, $0.025 \mathrm{ml}$ of $0.33 \%$ red blood cell suspension was added and the mixture incubated at $37^{\circ} \mathrm{C}$ for $1 \mathrm{hr}$. The result was considered positive when all red blood cells precipitated on the bottom of the well. The end point dilution of the positive antibody was determined to be the $\mathrm{HI}$ antibody titer. Each run had two positive and two negative controls.

RESULTS

Seroepidemiology of dengue virus infection in LiuChiu. The geographic distribution of eight villages on the island and their positive rates is shown in Figure 1. The MAC-ELISA data showed that $35(2.80 \%)$ of 1,232 blood samples were IgM-positive (Table 1). Statistical analysis did not show a significant correlation between the IgM positive rate and geographic distribution $(P>0.05$, by chi-square test), and their prevalence odds ratios were inconsistent (Table 1).

Analysis of age distribution of IgM-positive individuals showed that the highest prevalence odds ratio was only 2.05 among the 15-year olds and the lowest was among the 13-year olds. We did not see the correlation of age with the prevalence rate $(P>0.05$, by chi-square test) probably because all sera were collected from persons with a very narrow age range (8-15 years of age).

The HI antibody test with 35 IgM-positive individuals showed that 20 had titers $<1: 10(57.14 \%)$, with the others having $\mathrm{HI}$ titers ranging from 1:10 to $>1: 80$ (Table 2).

Seroepidemiology in Tung-Kang. Sixteen of 925 sera $(1.73 \%)$ collected in 1991 from three elementary schools in Tung-Kang were IgM-positive (Table 3 ). The prevalence rates among three schools were significantly different $(P<$ 0.01 , by chi-square test). However, only two of another 192 community-based serum specimens were IgM positive (Table 3).

Seroepidemiology in the Kaohsiung area. Fifty-seven blood samples were collected from suspected cases in Harn's Clinic and at the Kaohsiung Medical College Hospital. Those collected in 1991 were all negative while one of 34 sera collected in 1992 and one of 17 collected in 1993 (January-July) were positive.

A total of 642 serum samples were collected from three districts in the Kaohsiung area, i.e., Kang-San, Feng-San, and San-Min. Four $(0.62 \%)$ were determined to be IgMpositive (Table 4). Statistical analysis showed that location was not sigificantly correlated with the prevalence rate in this area $(P>0.05$, by chi-square test).

\section{DISCUSSION}

In the present study, IgM antibody was detected in both clinical and nonclinical community sera, indicating that the MAC-ELISA can be a useful tool for antibody screening. ${ }^{12.19,22-25}$ The technique has also been proven to be superior to the HI test not only in sensitivity but also in specificity. ${ }^{14}$ It was even more useful with outpatients in the community because it does not require a second blood specimen as with the $\mathrm{HI}$ test. This versatile serologic technique has been extensively used in detecting dengue antibodies both in epidemic and nonepidemic areas. ${ }^{26}$

In this study, more than half of the IgM positive samples were HI-negative. Because most dengue cases in Taiwan are primary infections, the HI antibody (IgG) titer of some serum samples seems to have been too low to be detected by 


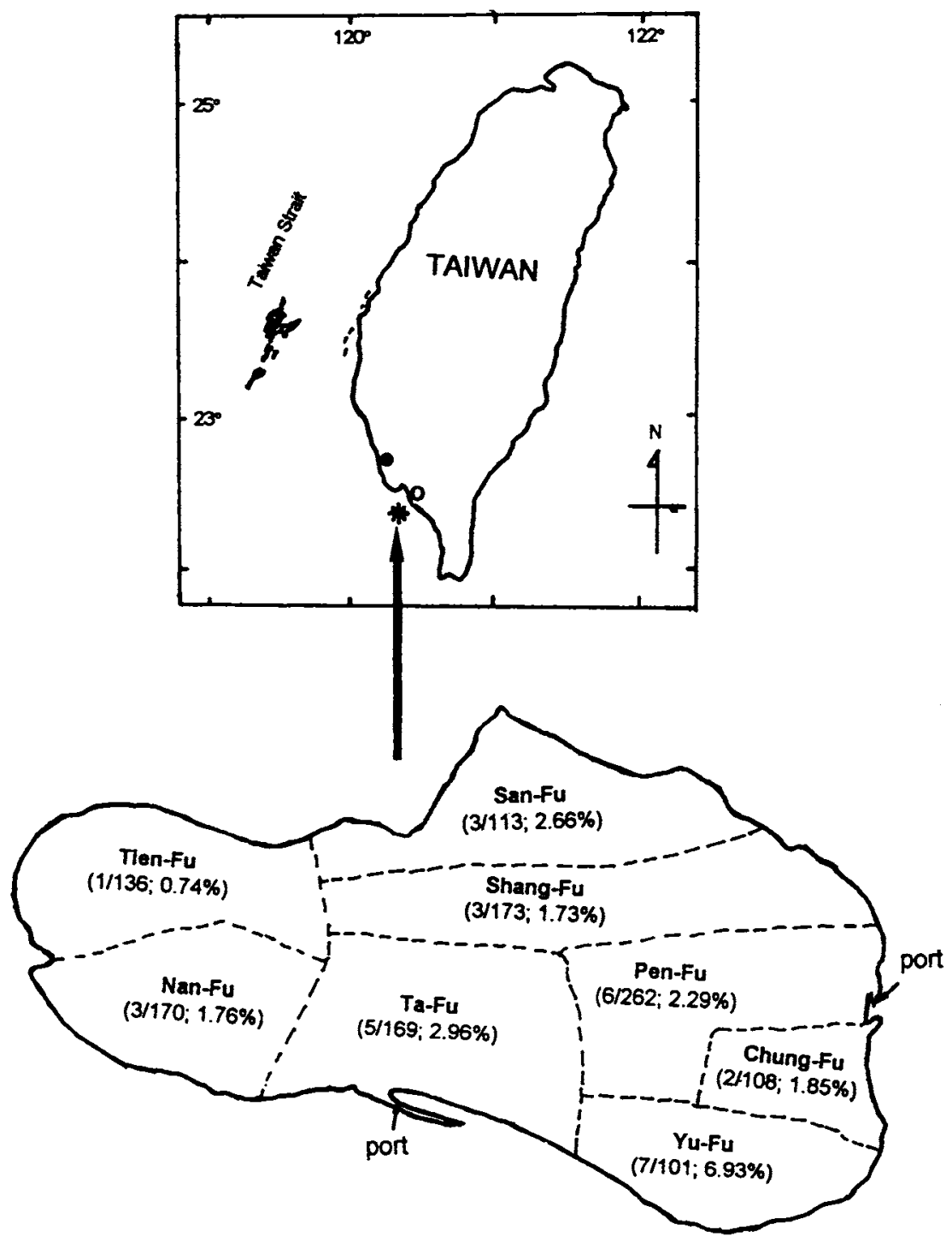

FIGURE 1. Map and IgM antibody distribution among eight villages in Liu-Chiu. $\bullet=$ Kaohsiung; $0=$ Tung-Kang; $*=$ Liu-Chiu.

the HI test at the time when IgM antibody appears in the blood.

In southern Taiwan, dengue fever has appeared intermittently since the 1987-1988 epidemic. Two outbreaks causing 149 and 220 confirmed indigenous cases were reported in 1991 and 1994, respectively. ${ }^{27}$ Inapparent or subclinical in-

TABLE 1

Geographic distribution of IgM antibody in Liu-Chiu, Taiwan, 1990*

\begin{tabular}{lcc}
\hline \multicolumn{1}{c}{ Village } & $\begin{array}{c}\text { No. positive/no. examined } \\
(\%)\end{array}$ & $\begin{array}{c}\text { Prevalence odds } \\
\text { ratio }+\end{array}$ \\
\hline Nan-Fu & $3 / 170(1.76)$ & 2.38 \\
Ta-Fu & $5 / 169(2.96)$ & 4.00 \\
Yu-Fu & $7 / 101(6.93)$ & 9.36 \\
Chung-Fu & $2 / 108(1.85)$ & 2.50 \\
Pen-Fu & $6 / 262(2.29)$ & 3.09 \\
Shang-Fu & $3 / 173(1.73)$ & 2.34 \\
San-Fu & $3 / 113(2.66)$ & 3.59 \\
Tien-Fu & $1 / 136(0.74)$ & 1 \\
\hline
\end{tabular}

$x^{2}=11.35$, degrees of freedom $=7, P>0.05$

$\uparrow$ positive in each village/\% positive in Tien-Fu. fection seems to be more common than was expected. ${ }^{10}$ The risk of emerging dengue hemorrhagic fever/dengue shock syndrome (DHF/DSS) prompted us to conduct this investigation.

The continued transmission of dengue virus was observed in Liu-Chiu. Although prevalence rates among eight villages did not show a significant difference, the prevalence-odds ratio in $\mathrm{Yu}-\mathrm{Fu}$ was as high as 9.36 when compared with

TABLE 2

Relationships between hemagglutination inhibition $(\mathrm{HI})$ antibody titers and IgM-positive samples among sera collected from LiuChiu, Taiwan, 1990

\begin{tabular}{cc}
\hline HI titer & No. IgM positive (क) \\
\hline$<1: 10$ & $20(57.14)$ \\
$1: 10$ & $2(5.71)$ \\
$1: 20$ & $4(11.43)$ \\
$1: 40$ & $8(22.86)$ \\
$>1: 80$ & $1(2.86)$ \\
Total & $35(100)$ \\
\hline
\end{tabular}


TABLE 3

IgM antibody distribution among three elementary schools in TungKang, Taiwan, 1991*

\begin{tabular}{lc}
\hline School & No. positive examined (\%) \\
\hline Tung-Kang & $3 / 316(0.95)$ \\
Hai-Pin & $7 / 144(4.86)$ \\
Tung-Long & $6 / 465(1.29)$ \\
\hline
\end{tabular}

${ }^{*} \chi^{2}=13.98$, degrees of freedom $=2, P<0.01$.

Tien-Fu, in which the prevalence rate was the lowest, suggesting that the dengue virus was relatively active in Yu-Fu. Villages located in areas close to ports have usually shown higher prevalence rates. Fishing boats were believed to be associated with introduction of the virus from neighboring endemic countries. In addition, crowded living conditions was also considered a risk factor for a higher rate of dengue infections. ${ }^{28}$ Thus, it was not surprising that $\mathrm{Yu}-\mathrm{Fu}$ had a higher prevalence rate since this village was the most crowded locality on the island. The virus may subsequently spread to adjacent agricultural villages. However, a vector control campaign has been conducted on the island by health authorities since $1987,{ }^{29}$ and eventually limited the spread of the virus. Illegal fishermen, mostly from adjacent countries, may in part account for the introduction of dengue virus. It was noted that Chung-Fu had a lower IgM antibody prevalence rate even though it is located adjacent to $\mathrm{Yu}-\mathrm{Fu}$ village, but it had a higher HI antibody titer rate (Chien LJ, National Yang-Ming Medical college, unpublished data).

The Tung-Kang survey revealed that the IgM prevalence rate is higher in school children than in adults from the community and was presumably associated with the high $\mathrm{HI}$ antibody rate in the adult population (Chien LJ, National YangMing Medical College, unpublished data). During the interview, many residents reported that they had had dengue fever in the previous years. Among three elementary schools in Tung-Kang, the school in Hai-Pin had the highest prevalence rate, probably because it was located in the proximity of the fishing port and most children from this school were from families with fishing as an occupation. The virus may have been introduced in the same way as that in Liu-Chiu.

The low IgM antibody prevalence rate in the Kaohsiung area could be the result of a campaign on vector control after the outbreak occurring in 1991. However, it was not possible to exclude the possibility of persistent virus transmission even though prevalence rate was so low. Three districts in the Kaohsiung area showed no difference in their prevalence rates for IgM antibody, probably because all environments in theses areas were similar.

The existence of IgM antibody in all five areas has demonstrated that the dengue virus may have established a means of silent transmission in southern Taiwan. Asymptomatic infections or symtomatic infections that are not diagnosed as dengue fever must have been occurring in these areas. The occurrence of intermittent outbreaks such as those in 1991 and 1994 are not surprising. The most important concern is the emergence of DHF/DSS, as has been reported in Sri Larka, India, New Caledonia, and Tahiti. ${ }^{30} \mathrm{~A}$ confirmed fatal case of DHF occurred in $1994^{31}$ This was the only case of DHF in Taiwan thus far. Therefore, it would be worthwhile to establish a surveillance system for early de-
TABLE 4

IgM antibody distribution of community-based sera collected from three districts in the Kaoshiung area of Taiwan, 1992*

\begin{tabular}{lc}
\hline District & No. positive/no. examined (क) \\
\hline San-Min & $2 / 205(0.98)$ \\
Kang-San & $1 / 203(0.49)$ \\
Feng-San & $1 / 234(0.43)$ \\
\hline
\end{tabular}

$x^{2}=0.06$, degrees of freedom $=2, P>0.05$.

tection as an indicator of epidemic potential. Active screening using the MAC-ELISA is suggested to be the best technique because of its high sensitivity and versatility.

Acknowledgment: We are indebted to Dr. Duane J. Gubler for kindly providing some of the reagents for the assays.

Financial support: This work was supported by a grant from Department of Health, Republic of China (DOH82-TD-059).

Authors' addresses: Wei-June Chen, Su-Lih Chen, and Jih-Hua Fang. Department of Parasitology. Chang Gung College of Medicine and Technology, Kwei-San. Tao-Yuan 333, Taiwan. Li-Jung Chien, Chi-Chen Chen, and Chwan-Chuen King, School of Public Health, National Taiwan University, Taipei 100. Taiwan. Min-Rong Harn, Harn's Clinic, Kaohsiung 807, Taiwan. Kao-Pin Hwang, Department of Pediatrics, Kaohsiung Medical College, Kaoshiung 807, Taiwan.

Reprint requests: Wei-June Chen, Department of Parasitology, Chang Gung College of Medicine and Technology, Kwei-San, TaoYuan 333, Taiwan.

\section{REFERENCES}

1. Halstead SB, 1980. Dengue haemorthagic fever-a public health problem and a field for research. Bull World Health Organ 58: $1-21$.

2. Goh KT, Ng SK, Chan YC, Lim SJ, Chua EC, 1987. Epidemiological aspects of an outbreak of dengue fever/dengue haemorrhagic fever in Singapore. Southeast Asian J Trop Med Public Health 18: 291-294.

3. Wu YC, 1986. Epidemic of dengue 2 on Liou-Chyou Shiang, Pingtung, Taiwan, in 1981. Chin J Microbiol Immunol 9: 203-211.

4. Hsieh WC, Chen MF, Lin KT, Hsu ST, Ma CI, Wu SS, 1982. Study of outbreak of dengue fever on Liou-Chyou Shiang, Ping-Tung county in 1981. I Formos Med Assoc 81: 13881395.

5. Ko YC, 1989. Epidemiology of dengue fever in Taiwan. Kaohsiung J Med Sci 5: 1-11.

6. Department of Health, Executive Yuan, ROC, 1987. Preliminary report of the dengue investigation in Taiwan area. Epidemiol Bull 3: 93-99.

7. Department of Health, Executive Yuan, ROC, 1989. Epidemiology of dengue in Taiwan area. Epidemiol Bull 5: 1-3.

8. Gubler DJ, 1987. Current research on dengue. Harris KF, ed. Current Topics in Vector Research. Volume 3. New York: Springer-Verrlag, 37-56.

9. Huang JS, Hsu EL, 1984. Investigations on the distribution and breeding habitats of dengue vectors in Kaohsiung city. Chin J Entomol 14: 233-244.

10. Malison MD, Waterman SH, 1983. Dengue fever in the United States. JAMA 249: 496-500.

11. Nogueira RMR, Schatzmayr HG, Miagostovich MP, Fatima $M$, Fatima DB, Filho JCF, 1988. Virological study of a dengue type 1 epidemic at Rio de Janeiro. Mem Inst Oswaldo Cruz 23: 219-225.

12. Innis BL, Nisalak A, Nimmannitya $S$, Kusalerdchariya $S$, Chongswadi V, Suntayakorn S, Puttisri P, Koke CH, 1989. An enzyme-linked immunosorbent assay to characterize dengue infection where dengue and Japanese encephalitis co-circulate. Am J Trop Med Hyg 40: 418-427. 
13. Burke DS, Nisalak A, 1982. Detection of Japanese virus immunoglobulin $M$ antibodies in serum by antibody capture radioimmunoassay. J Clin Microbiol 15: 353-361.

14. Chen WJ, 1989. Serological and virological diagnosis for dengue infection. Kaohsiung J Med Sci 5: 66-71.

15. Botros BAM, Watts DM, Soliman AK, Salib AW, Moussa MI, Mursal H, Douglas C, Farah M, 1989. Serological evidence of dengue fever among refugees, Hargeysa, Somalia. $J$ Med Virol 29: 79-81.

16. Chow L, Hsu ST, 1989. MAC-ELISA for the detection of IgM antibodies to dengue type 1 virus. Chin J Microbiol Immunol 22: 278-285.

17. Chen WJ, Fang AH, Liaw YH, Lo FL, Wei YY, Ko YC, Liu WT, Chow HR, King CC, Kao CL, Yang HY, Chuang $\mathrm{CH}$, Wu YC, Chou MJ, Chen CJ, 1990. Antibody response by MAC-ELISA to dengue viruses during the epidemic in southern Taiwan, 1988. Arbovirus Res Aust 5: 35-36.

18. Chow L, Sun HC, Chen HY, Lin SY, Wu JS, 1992. Detection and differentiation of dengue- 1 from Japanese encephalitis virus infections by ABC MAC-ELISA. Chin J Microbiol Immunol 25: 172-180.

19. Kuno G, Gomez I, Gubler DJ, 1987. Detecting artificial antidengue IgM immune complexes using an enzyme-linked immunosorbent assay. Am J Trop Med Hyg 36: 153-159.

20. Kuberski TT, Rosen L, 1977. A simple technique for the detection of dengue antigen in mosquito by immunofluorescence. Am J Trop Med Hyg 26: 533-537.

21. Clarke DH, Casals J, 1958. Techniques for hemagglutination and hemagglutination-inhibition with arthropod-borne viruses. Am J Trop Med Hyg 7: 561-573.

22. Chen WJ, Hwang KP, Fang AH, 1991. Detection of IgM antibodies from cerebrospinal fluid and sera of dengue fever pa- tients. Southeast Asian J Trop Med Public Health 22: 659 663.

23. Bundo K, Igarashi A, 1985. Antibody capture ELISA for detection of immunoglobulin $M$ antibodies in sera from Japanese encephalitis and dengue hemorrhagic fever patients. $J$ Virol Methods 11: 15-22.

24. Nogueira RMR, Miagostovich MP, Cavalcanti SMB, Marzochi KBF, Schatzmayr HG, 1992. Levels of IgM antibodies against dengue virus in Rio de Janeiro, Brazil. Res Virol 143: 423427.

25. Zeller HG, Traore-Lamizana M, Monlum E, Hervy JP, Mondo $M$, Digoutte JP, 1992. Dengue-2 virus isolation from humans during an epizootic in southeastern Senegal in November, 1990. Res Virol 143: 101-102.

26. Gubler DJ, 1989. Surveillance for dengue and dengue hemorrhagic fever. Bull Pan Am Health Organ 23: 397-404.

27. Lee SF, Chou HC, Wu PH, Wu YC, Chou L, 1995. Investigating a dengue fever outbreak in Fenshan city of Kaohsiung County. Epidemiol Bull 11: 49-57.

28. Morens DM, Rigau-Perez JG, Lopez-Correa RH, Morre CG, Ruiz-Tiben EE, Sather GE, Chiriboga J, Eliason DA, CastaVelez A, Woodall JP, 1986. The dengue outbreak investigation group: dengue in Puerto Rico, 1977: public health response to characterize and control an epidemic of multiple serotypes. Am J Trop Med Hyg 35: 197-211.

29. Wang CH, Hwang JS, Lay JR, 1990. Preliminary study on the biological control of dengue vectors by fish in Liou-Chyou prefecture, Ping-tung County, Taiwan. Kaohsiung J Med Sci 6: 382-388.

30. Lam SK, 1990. Dengue and dengue hemorrhagic fever. Southeast Asian J Trop Med Public Health 21: 520-521.

31. Hsu YT, Huang PJ, Chen KH, Su CY, Chuang CH, Hung HY, Fang RN, Lu PH, 1994. Dengue hemorthagic fever: a case report. Epidemic Rep 10: 259-260. 